\title{
Mechanisms of atmospheric aerosols using mathematical modelling
}

\author{
P. Meenapriya ${ }^{1 *}$
}

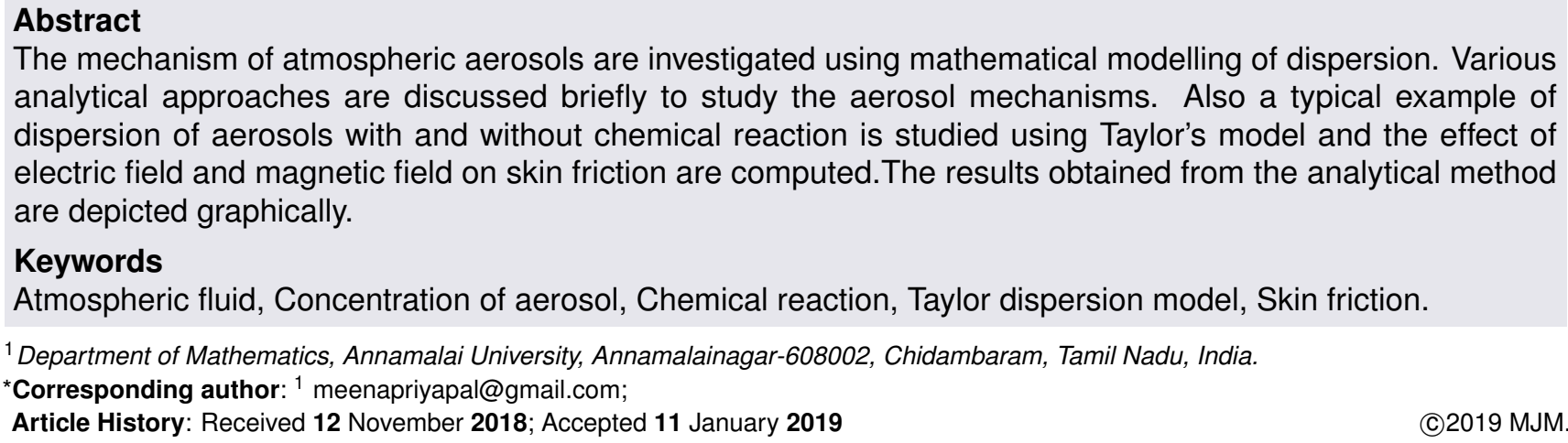

\section{Contents}

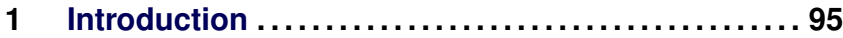

2 Typical example ............................. 96

3 Skin friction................................97

4 Results and Discussion ....................... 97

5 Conclusion ................................... 98

References $\ldots \ldots \ldots \ldots \ldots \ldots \ldots \ldots \ldots \ldots \ldots \ldots, 98$

\section{Introduction}

The Earth's atmosphere can be considered as a diluted complex aerosol system and such the impact of atmospheric aerosols is felt in all aspects of our life. The aerosols have potential to warm or cool the earth's atmosphere system depending upon their absorptivity and cloud albedo. Atmospheric aerosols are complex mixtures of different chemical species. In the upper atmosphere, the presence of aerosols can significantly decrease the temperature, thus contributing to the overall greenhouse effect and global climate. The size of the atmospheric aerosol particles can range from 1 nanometer $(\mathrm{nm})$ to $100 \operatorname{micrometers}(\mu \mathrm{m})$ in diameter. Several reports are available for the dynamics of aerosols over the globe as studied in $[10,11,12,13]$.

They can be emitted directly to the atmosphere (primary particles) or arise by gas-to-particle formation from precursor gases (secondary particles). The coarse fraction particles are mostly injected directly to the atmosphere by mechanical processes or from natural sources. The fine fraction contains mainly particles, which arise from gas-to-particle conversion. It can affect the climate in different ways.

1.1 Importance of the study of dispersion of aerosols

Pollutant transport resulting from municipal, industrial and agricultural wastes polluting atmosphere where control of pollutants is desired. The aerosols which are gradually choking people to death, where an understanding of the spreading of aerosols is required.

In view of above theoretical study on the effect of chemical reaction,size of aerosols and deformation of aerosols on dispersion is needed.Many mathematical models are developed on primary and secondary aerosols in the atmosphere as studied in $[14,16,17]$. The objective of this paper is to present the mechanisms of dispersion of aerosols in the atmosphere with and without chemical reaction under the effect of electric and magnetic field with a typical example.

\subsection{Literature survey}

The work on dispersion phenomena is fairly well known as in $[1,8,9]$ and others were mainly valid for large time after injecting a slug into the fluid. Later, dispersion was generalized it to cover transient phenomena, called a generalized dispersion model given in [6].

1.3 Dispersion models

The following analytical approaches are used to study the dispersion models with and without chemical reaction.

1.3.1 Analytical Approaches

1.Taylor dispersion model 
A simple method for the calculation of contaminant dispersion in bounded shear flow at long interval of time after injection is studied in [9]. He noted that solute is much more slowly dispersed by molecular diffusion across a velocity shear.

2.Aris Approach

The moment approach to generalize Taylor's result to include the effect of axial molecular diffusion Aris calculated the first few integral moments of a solute as it disperses in [1]. He obtained from the second moment,the dispersion coefficient for a solute with molecular diffusivity injected into a tube of radius a containing fluid in a laminar flow with mean speed $\mathrm{u}$. 3.Generalised dispersion model

The generalised dispersion model was developed and later the dispersion in a clear fluid was studied in [2,3] and this model was developed to study dispersion in porous media which is valid for all time as in [8].

4.Lighthill Approach

An analytical solution for the unsteady convective diffusion equation using an asymptotic expansion was investigated as in [7]. Later Lighthill's results was extended in [4] following the approach in [15].

5.Randomwalk approach

In the randomwalk approach, the tracer particles are represented by a large number of marked particles, whose paths are traced as they move through the fluid. In the next section one example dealing with dispersion of aerosols in the atmosphere using [9] is discussed.

\section{Typical example}

A mathematical model is developed to study the dispersion of aerosols with and without chemical reaction in the presence of electric and magnetic field using [9].

\subsection{Mathematical Formulation}

Consider the laminar flow through a horizontal parallel plates embedded with electrodes located at $\mathrm{y}=0$ and $\mathrm{y}=\mathrm{h}$ with an applied magnetic field $B_{o}$.

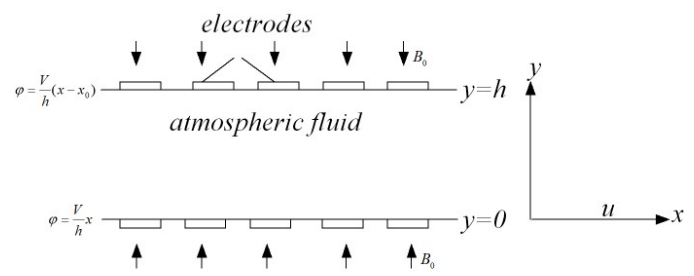

Figure 1. Physical Configuration

The momentum and concentration equations are given by,

$$
\frac{d^{2} u}{d y^{2}}-M^{2} u=W e \rho_{e} E_{x}+P
$$

where $\mathrm{u}$ is the velocity, We is the electric number, $\mathrm{P}$ is the pressure.

$$
\frac{\partial C}{\partial t}+u \frac{\partial C}{\partial x}=D \frac{\partial^{2} C}{\partial y^{2}}-K C
$$

where $P=\frac{\partial p}{\mu \partial x}, \mu$ is the Coefficient of viscosity .

The solution of equation(2.1) satisfying the conditions $u=0$ at $y=0,1$ is

$$
u=A \cosh M y+B \sinh M y+\frac{W_{e} a_{0} e^{-\alpha y}}{\left(\alpha^{2}-M^{2}\right)}-\frac{P}{M^{2}}
$$

where $\alpha$ is the volumetric coefficient for conductivity. where $a_{0}=\frac{W_{e} x_{0} \alpha^{2}}{e^{-\alpha}-1} ; A=\frac{P}{M^{2}}-\frac{W_{e} a_{0} e^{-\alpha y}}{\left(\alpha^{2}-M^{2}\right)}$;

$$
B=\frac{1}{\sinh M}\left(\frac{P}{M^{2}}(1-\cosh M)-\frac{W_{e} a_{0} e^{-\alpha y}}{\left(\alpha^{2}-M^{2}\right)}\left(e^{-\alpha}-\cosh M\right)\right) ;
$$

From equation(2.3) the average velocity is,

$$
\begin{array}{r}
\bar{u}=\int_{0}^{1} u d y=\frac{A \sinh M}{M}-\frac{B \cosh M-1}{M}+ \\
\frac{W_{e} a_{0}}{\alpha\left(\alpha^{2}-M^{2}\right)}\left(e^{-\alpha}-1\right)-\frac{P}{M^{2}}
\end{array}
$$

Since considering advection across a plane moving with the mean speed of flow, the fluid velocity relative to this plane is given by

$$
V=u-\bar{u}=A \cosh M y+B \sinh M y+\frac{W_{e} a_{0} e^{-\alpha y}}{\left(\alpha^{2}-M^{2}\right)}+f
$$

where $f=-\frac{A \sinh M}{M}-\frac{B \cosh M-1}{M}+\frac{W_{e} a_{0}}{\alpha\left(\alpha^{2}-M^{2}\right)}\left(e^{-\alpha}-1\right)$ using equation(2.5) and the non-dimensional quantities $y^{*}=\frac{y}{h} ; C^{*}=\frac{C}{C_{0}} ; t^{*}=\frac{t}{\bar{t}} ; \xi=\frac{x-\bar{u} t}{L} ; \beta^{2}=\frac{h^{2}}{D} K$ and following Taylor(1953),assuming partial equilibrium equation(2.2) takes the form

$$
\frac{\partial^{2} C}{\partial y^{2}}-\beta^{2} C=Q V
$$

where $Q=\frac{h^{2}}{D L} \frac{\partial C}{\partial \xi}$ and $\beta$ the reaction rate parameter.

We consider two cases. In case 1 in the presence of chemical reaction and the case 2 without chemical reaction.

\section{Case 1: With Chemical reaction $(\beta \neq 0)$}

The solution of equation(2.6), using (2.5) and the conditions $\frac{\partial C}{\partial y}=0$ at $y=0$

and $C=1$ at $y=1$ is

$$
\begin{aligned}
C= & Q\left(C 1 \cosh \beta y+C 2 \sinh \beta y+\frac{A \cosh M y}{M^{2}-\beta^{2}}+\frac{B \sinh M y}{M^{2}-\beta^{2}}\right. \\
& \left.+\frac{W_{e} a_{0}}{\left(\alpha^{2}-M^{2}\right)} \frac{e^{-\alpha y}}{\left(\alpha^{2}-\beta^{2}\right)}-\frac{f}{\beta^{2}}\right)
\end{aligned}
$$

$$
\begin{aligned}
C 1= & \frac{1}{\cosh \beta}\left(1-\frac{B}{M^{2}-\beta^{2}}\left(\frac{-M \sinh \beta}{\beta}+\sinh M\right)-\right. \\
& \frac{W_{e} a_{0}}{\left(\alpha^{2}-M^{2}\right)\left(\alpha^{2}-\beta^{2}\right)}\left(\frac{\alpha \sinh \beta}{\beta}+e^{-\alpha}+\frac{f}{\beta^{2}}\right) ;
\end{aligned}
$$

$$
C 2=\frac{1}{\beta}\left(-\frac{B M}{M^{2}-\beta^{2}}+\frac{\alpha W_{e} a_{0}}{\left(\alpha^{2}-M^{2}\right)\left(\alpha^{2}-\beta^{2}\right)}\right)
$$


Case 2: Without chemical reaction $(\beta=0)$

when $\beta=0$, equation(2.6) becomes

$$
\frac{\partial^{2} C_{0}}{\partial y^{2}}=Q_{0} V
$$

where $C_{0}$ is the concentration of aerosols without chemical reaction and $Q_{0}=\frac{h^{2}}{D L} \frac{\partial C_{0}}{\partial \xi}$ The solution of equation (2.8) using (2.5)is

$$
\begin{aligned}
C_{0}= & Q\left(\frac{A \cosh M y}{M^{2}}+\frac{B \sinh M y}{M^{2}}+\frac{W_{e} a_{0} e^{-\alpha y}}{\alpha^{2}\left(\alpha^{2}-M^{2}\right)}\right. \\
& \left.-\frac{f y^{2}}{2}\right)+f 1 y+f 2,
\end{aligned}
$$

where $\mathrm{f} 1$ and $\mathrm{f} 2$ are constants to be determined using the entry condition.

2.2 Taylor's Diffusion co-efficient

The volumetric rate at which the solute is transported across a section of the channel of unit breadth is

$$
\begin{aligned}
& M_{1}=\int_{0}^{1} C V d y \\
& M_{2}=\int_{0}^{1} C_{0} V d y
\end{aligned}
$$

using equations(2.7)and(2.5) performing the integration and after simplification we get,

$$
\begin{gathered}
M_{1}=\frac{h^{3}}{D L} G_{1} \frac{\partial C_{m}}{\partial \xi} \\
M_{2}=\frac{h^{3}}{D L} G_{2} \frac{\partial C_{m}}{\partial \xi} \\
\text { where }^{*}=-\frac{h^{3}}{2 D} G_{1} \\
D_{1}^{*}=-\frac{h^{3}}{2 D} G_{2}
\end{gathered}
$$

The fact that no material is lost in the process is expressed by the continuity equation for $C_{m}$ namely

$$
\frac{\partial M}{\partial \xi}=-\frac{2}{L} \frac{\partial C_{m}}{\partial t}
$$

equation(2.16)using (2.12) and (2.13) becomes

$$
\begin{aligned}
& \frac{\partial C_{m}}{\partial t}=D^{*} \frac{\partial^{2} C_{m}}{\partial \xi^{2}} \\
& \frac{\partial C_{m}}{\partial t}=D_{1}^{*} \frac{\partial^{2} C_{m}}{\partial \xi^{2}}
\end{aligned}
$$

which is the equation governing the longitudinal dispersion. The lengthy expression of dispersion co-efficient's $D^{*}, D_{1}^{*}$ are computed and the results are discussed in the next section.

\section{Skin friction}

In many practical applications it is advantageous to know the skin friction at the boundaries. It can be determined once we know the velocity. The skin friction $\tau$ at the walls is defined as

$$
\tau=\mu\left(\frac{\partial u}{\partial y}\right)_{y= \pm h}
$$

making this dimensionless using the scale for u used earlier we get,

$$
\tau=\left(\frac{d u}{d y}\right)_{y= \pm 1}
$$

Here $\left(\frac{d u}{d y}\right)$ can be obtained using (2.3). The analytical results obtained are displayed through graphs.

\section{Results and Discussion}

A mathematical modelling of dispersion of atmospheric aerosols is discussed using Taylor's model. The analytical results are reported and are displayed graphically through figures.

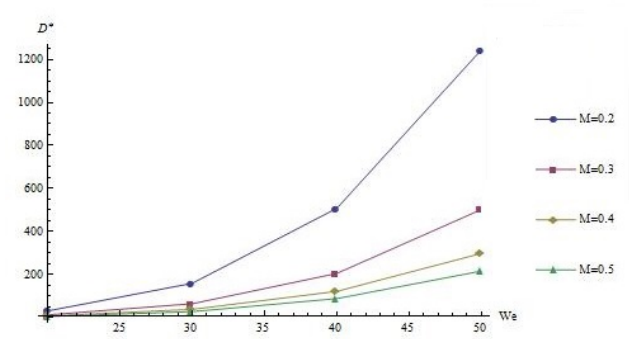

Figure 2. Dispersion coefficient $D^{*}$ with Electric number We for different values of Hartmann number $\mathrm{M}$ for $\beta=1$

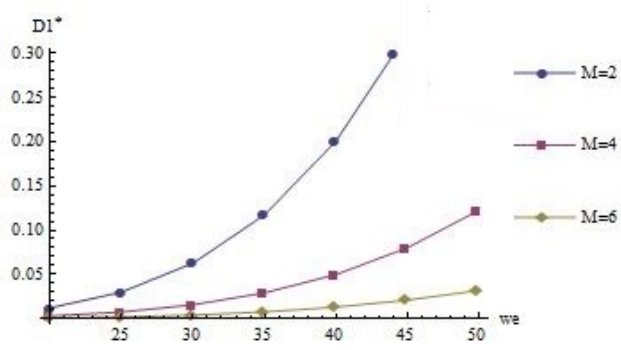

Figure 3. Dispersion coefficient $D_{1}^{*}$ with Electric number We for different values of Hartmann number for $\beta=0$

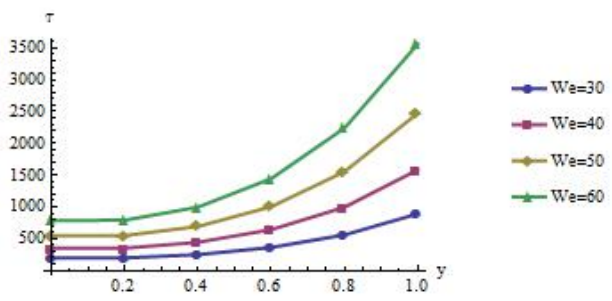

Figure 4. Skin friction coefficient $\tau$ with y for different values of Electric number We 


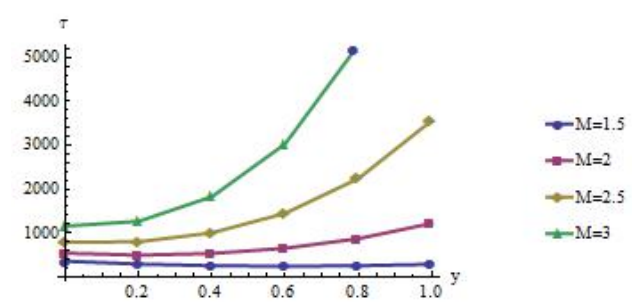

Figure 5. Skin friction coefficient $\tau$ with y for different values of Hartmann number M

Figure 2 reveals that the dispersion coefficient $D^{*}$ increases with an increase in electric number We and decreases with increase in Hartmann number M. Figure 3 shows that the dispersion coefficient $\mathrm{D} 1 *$ increases with increases in electric number We and decreases with increase in Hartmann number M.From the figures 4 and 5,it is seen that the enhancement of electric and magnetic field increases the skin friction. It conclude that the presence of electric field and magnetic field enhances the dispersion of aerosols for both with and without chemical reaction. It suggests that the dispersion of aerosols and skin friction would depend upon the parameters in the atmosphere.

\section{Conclusion}

The Mechanisms of dispersion of atmospheric aerosols using mathematical modelling is investigated. Various theoretical approaches available on dispersion are discussed. The problem considered in this paper are representative of aerosol mechanisms in the atmosphere. It conclude that there is impact of electric field and magnetic field on dispersion of aerosols for both with and without chemical reaction.

\section{References}

[1] R. Aris, On the dispersion of a solute in a fluid flowing through a tube, Proc.R.Soc.London, 235(1956), 67-77.

[2] N. G. Barton, On the method of moments for solute dispersion, J. Fluid Mech., 126(1983), 205-218.

[3] N. G. Barton and A. N. Strokes, A computational method for sheared dispersion in parallel flows, In. Comp. Tech. App., 85(1986), 345-355.

[4] P. C. Chatwin, On the Interpretation of some longitudinal dispersion experiments, J. Fluid Mech., 48(1971), 689702.

[5] P. C. Chatwin, Longitudinal dispersion of dye whose concentration varies Harmonically with time, J.Fluid Mech., 58(1973), 657-667.

[6] W. N. Gill and R. Sankarasubramanian, Exact analysis of unsteady convective diffusion, Proc.R.Soc.London, 316(1970), 341-350.

[7] M. J. Lighthill, Motion in narrow capillaries from the standpoint of lubrication theory in circulating and respiratory mass transport, G.E.N. Wolstenholme and J.Knight (eds) (1969), 85-109.
[8] P. N. Shivakumara, N. Rudraiah, D. Pal, and P.G. Siddheshwar, Closed form solution for unsteady convective diffusion in a fluid saturated sparsely packed porous medium, Int. Commun. Heat Mass Transfer, 14(1987), 137-145.

[9] G. I. Taylor, Dispersion of solute matter in solvent flowing through a tube, Proc. R. Soc. London. Ser.A, 219(1953), 186-203.

[10] F. Raes, T. Bates, F. Mc. Govern and M. VanLiedeKereke, The second aerosol characterization experiment(ACE-2), General Overview and Main Results,T ellus., 52(2000), $1-11$.

[11] D. Muller, K. Franke, F. Wagner, D. Althousen, A. Ansmann, and J. Heintzenberg, Vertical profiling of optical and physical particle properties over the tropical Indian ocean with six wavelengths lidar, J. Geophys. Res., 106(D22)(2001), 28567-28575.

[12] K. Niranjan, B. L. Mahavan, and V. Srekanth, Micropulse lidar observation of high altitude aerosol layers at vishakapatnam located on the east coast of india,Geophys, Res. Let., 34(2007), L03815.

[13] S. K. Satheesh, V. Vinoj and K. Krishnamoorthy, Vertical distribution of aerosols over an urban continental site in India inferred using a micropulse lidar, Geophys.Res. Let., 33(20)(2006), L20816.

[14] N. Satheesh and C. O. Ng, Dispersion in porous media with and without chemical reaction:A review, Journal of Porous Media, 10(3)(2007), 219-248.

[15] P. G. Saffman, On the boundary condition at the surface of a porous medium, Stud. Appl. Math., 50(1971), 93101.

[16] P. Meenapriya, Dispersion of aerosols in atmospheric fluid flow, International Journal of Soft Computing, Mathematics and Control, 4(2015), 2-13.

[17] N. Devaraju, Mathematical Modelling of Dispersion of Atmospheric Aerosols, Thesis, Bangalore University, 2009. 